Article

\title{
Characterizing Motif Dynamics of Electric Brain Activity Using Symbolic Analysis
}

\author{
Massimiliano Zanin ${ }^{1,2}, *$ and David Papo ${ }^{3}$ \\ ${ }^{1}$ Faculdade de Ciências e Tecnologia, Departamento de Engenharia Electrotécnica, Universidade Nova \\ de Lisboa, 2829-516 Caparica, Lisbon, Portugal \\ ${ }^{2}$ Innaxis Foundation \& Research Institute, José Ortega y Gasset 20, 28006 Madrid, Spain \\ ${ }^{3}$ Center for Biomedical Technology, Technical University of Madrid, Campus Montegancedo, 28223 \\ Pozuelo de Alarcón, Madrid, Spain; E-Mail: papodav@gmail.com
}
* Author to whom correspondence should be addressed; E-Mail: mzanin@innaxis.org;
Tel./Fax: +34-91-524-57-94.

External Editor: Osvaldo Anibal Rosso

Received: 9 September 2014; in revised form: 15 October 2014 / Accepted: 23 October 2014 /

Published: 27 October 2014

\begin{abstract}
Motifs are small recurring circuits of interactions which constitute the backbone of networked systems. Characterizing motif dynamics is therefore key to understanding the functioning of such systems. Here we propose a method to define and quantify the temporal variability and time scales of electroencephalogram (EEG) motifs of resting brain activity. Given a triplet of EEG sensors, links between them are calculated by means of linear correlation; each pattern of links (i.e., each motif) is then associated to a symbol, and its appearance frequency is analyzed by means of Shannon entropy. Our results show that each motif becomes observable with different coupling thresholds and evolves at its own time scale, with fronto-temporal sensors emerging at high thresholds and changing at fast time scales, and parietal ones at low thresholds and changing at slower rates. Finally, while motif dynamics differed across individuals, for each subject, it showed robustness across experimental conditions, indicating that it could represent an individual dynamical signature.
\end{abstract}

Keywords: motifs; entropy; forbidden patterns; electroencephalogram (EEG); time scales 


\section{Introduction}

Over the last twenty years, advances both in computational techniques and in non-linear dynamics have fostered a revolution in neuroscience. For the first time, it has been possible to analyze the functional connectivity of the brain, i.e., how pairs of brain regions collaboratively compute information, by analyzing how their respective dynamics are related. More recently, it has been realized that connectivity patterns form networks with structure at all scales, and whose properties are to some extent independent of the identity of its component nodes and edges [1].

Of the aspects of network structural properties that most attention have received in the last years, a special position should be granted to motifs. Motifs are patterns of connectivity involving a small number of nodes, occurring with a significantly higher frequency than expected in randomized networks [2]. Networks of synaptic connections between neurons have been shown to exhibit network motifs. Neural circuitry has been shown to be characterized by triplets of neurons connected as feedforward loops. For instance, it has been shown that the synaptic network of Caenorhabditis elegans shows a wide repertoire of motifs [3]. Electrical measurements of neuron tetrads showed that mammalian neuronal networks also display significant network motifs [4,5].

Studies of genetic regulatory networks suggest that motifs may be associated with specific functions, as memories or filters [6-8]. Likewise, neural motifs have been associated with an efficient integration of information [3], and simulations suggest that they may form the building blocks of fundamental aspects of brain dynamics and function [9].

Reference [3] proposed a distinction between structural and functional motifs, wherein structural motifs form the physical substrate for a repertoire of distinct functional modes of information processing that can potentially engage in a context-spefic fashion in different patterns of interactions [3]. Non-invasive electro or magnetoencephalographic recordings do not faithfully mirror the underlying anatomical circuits, so that the relationship between structural and functional networks is lost. While the interpretation of functional motifs in terms of computational units may then be lost, recent findings associating the presence of certain functional motifs with the appearance of pathological conditions [10] suggest that functional networks built using scalp-recorded EEG or MEG devices may still retain some important physiological meaning.

Motifs are typically constructed by averaging the connectivity of triplets of nodes over long time windows, This analysis disregards motifs' temporal evolution and only yields a single motif per triplet, so that motifs can only be counted. However, brain activity is characterized by the constant formation and destruction of couplings between distant brain regions. In the study of the brain, as well as of other real-world systems, motifs dynamics can encode important information that should not be discarded.

In this contribution, we propose a method for analyzing the coordinated dynamics of triplets of brain regions, which involves generalizing the concept of three-node motifs, and characterizing their temporal evolution using symbolic analysis.

\section{Method}

Given a set of $n$ time series $t_{i}(i \in[1, n])$, recorded by a set of $n$ sensors (e.g., electro- or magneto-encephalography, functional magnetic resonance imaging, etc.), which for the sake of 
simplicity, are suppose to all be of the same length, denoted by $l$, the first step in motif characterisation consists in selecting three sensors (nodes), i.e., $n_{1}, n_{2}$ and $n_{3}$. Notice that this process can be generalized to an arbitrary number of sensors (nodes), in order to obtain higher order motifs. The corresponding three time series $\left(t_{n_{1}}, t_{n_{2}}\right.$ and $\left.t_{n_{3}}\right)$ are divided into non-overlapping windows to length $\tau$-see the upper part of Figure 1 for a graphical example. These three fragments are then mapped into a motif, by calculating the synchronization level between pairs of sensors, and activating the corresponding link when such synchronization exceedes a threshold $\rho$. Notice that, at this point, the three time series are transformed into a sequence of motifs, which can be understood as a time evolving [11] or multi-layer network [12]. Finally, the time evolution of such motifs is analyzed by means of symbolic analysis. Specifically, each possible motif is associated to a symbol-bottom part of Figure 1. Several techniques are available to analyze the appearance frequency of such symbols [13-15]; here we use the following two methods:

(1) The Shannon entropy of the symbols succession, given by:

$$
E=-\sum_{i} p_{i} \log _{2} p_{i}
$$

$p_{i}$ being the probability of appearance of the $i$-th symbol.

(2) The number of forbidden motifs, i.e., the number of symbols that do not appear in the analyzed time series.

Figure 1. Graphical representation of the motif entropy estimation methodology. A triplet of raw time series (upper part) is divided into non-overlapping windows of length $\tau$. A linear correlation is then calculated between them, and a threshold $\rho$ is applied, in order to construct three-nodes subgraphs (middle part). Finally, a symbol is associated to each motif, thus transforming the original EEG data into a symbolic time series (lower part).

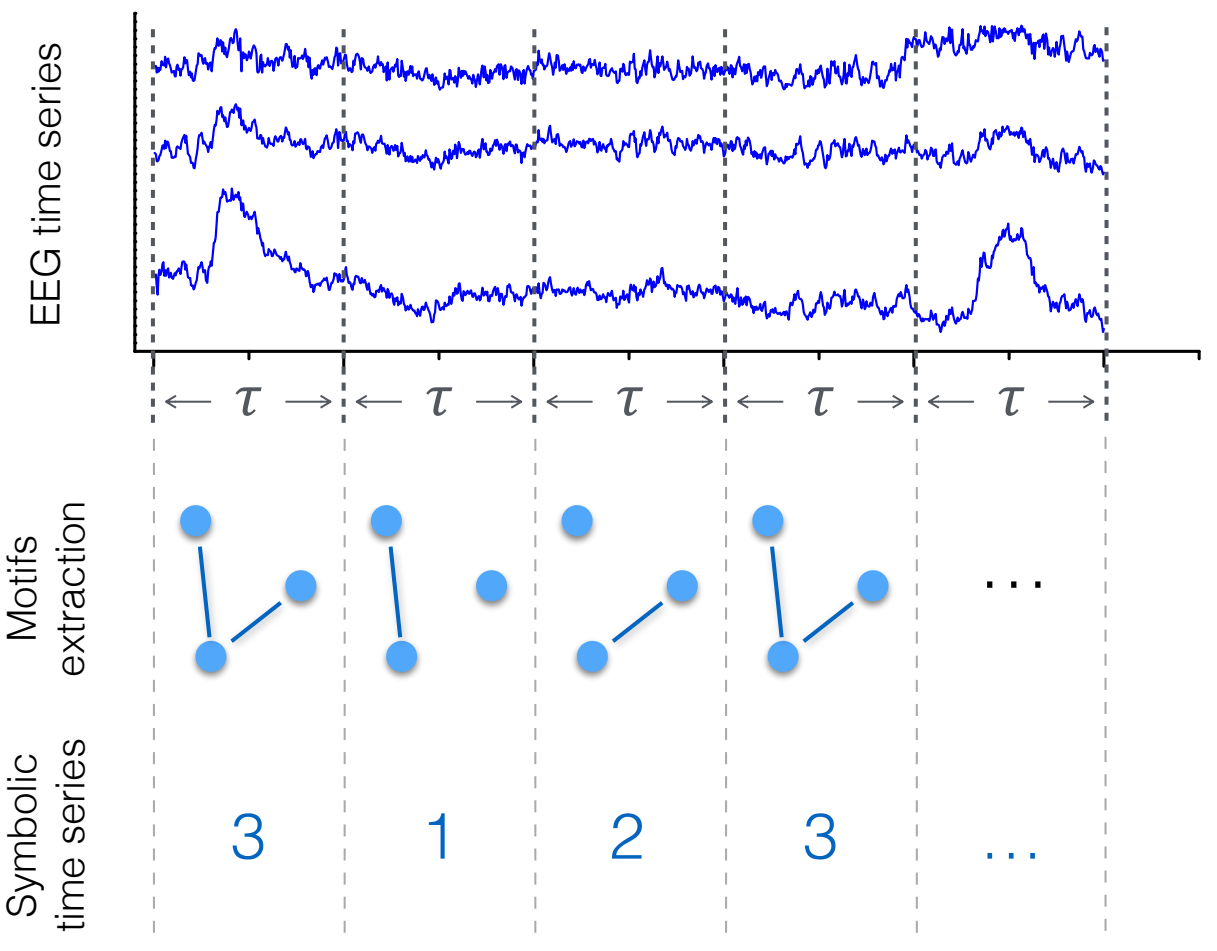


A high entropy, and consequently a low number of forbidden motifs, implies that the corresponding triplet of sensors is showing a high variability in its dynamics and connectivity pattern. On the other hand, the presence of a fixed motif, and thus of minimal entropy, suggests that the dynamics of the triplet does not evolve with time.

The goal ofthis methodology is ultimately two-fold. Firstly, by studying the evolution of the entropy as a function of the applied threshold $\rho$, it is possible to estimate the synchronization level that is optimal to obtain the richer dinamycs in the triplet. Secondly, an analysis of the entropy obtained for different time windows $\tau$ yields information about the characteristic time scale at which such dynamics develop.

Furthermore, the methodology presented here extends and merges two different approaches used in the study of brain dynamics. First, while in the original definition of a motif, its component nodes need to be connected [2], here we consider any possible pattern of connectivity among nodes, including disconnected graphs. Second, the analysis of the time evolution of motifs is performed in a way similar to the ordinal patterns methodology proposed by Bandt and Pompe for the analysis of univariate time series [16,17]. Instead of creating symbols from the evolution of a given observable through time, here each symbol represents the evolution through space, specifically through the space created by the different triplets of observables.

\section{Data Set Description}

Resting brain electrical activity was recorded from eight healthy volunteers under two conditions, eyes closed and open. Each condition lasted $24 \mathrm{~s}$. A standard 56 electrode montage, with electrodes positioned according to the extended $10-20$ System location, with a nasion reference was used. The electro-oculogram was also recorded, for blink, vertical, and horizontal eye movement correction [18]. The EEG was amplified $(0.05-100 \mathrm{~Hz})$ and digitized at $500 \mathrm{~Hz}$.

A Pearson's linear correlation was used to calculate the synchronization level between pairs of time series. While more complex metrics can be used, e.g., non-linear measures like Synchronization Likelihood [19], or causality measures like Granger Causality [20] or Transfer Entropy [21], here linear correlation was selected for its simplicity and low computational cost.

\section{Entropy and Thresholds}

Recalling that entropy represents the variability in the motifs within each triplet of sensors, an entropy close to zero implies that just one motif can appear. This is indeed the situation corresponding to extremely high (low) values of $\rho$, which always result in a completely disconnected (completely connected) motif. On the other hand, a high entropy should be expected between those extrema, indicating a rich neural dynamics.

Figure 2a depicts the evolution of the entropy as a function of the threshold applied for 5 triplets of nodes, for a large time window length $(\tau=60)$. It can be appreciated that a maximum is indeed observed, although for each one of the triplets this corresponds to a different $\rho$. Figure $2 \mathrm{~b}$ and $\mathrm{c}$ also respectively reports the histograms for the entropy and the optimal threshold for all the 175.616 triplets of sensors corresponding to a subject. Here, and in what follows, by optimal threshold we refer to the threshold that maximizes the motif entropy. 
Figure 2. Analysis of thresholds. (a) Evolution of the entropy of five triplets as a function of the threshold $\rho$, for a fixed window length $\tau=50$. (b) Histogram of the highest entropy obtained by optimizing $\rho$ for each triplet. (c) Histogram of the optimal $\rho$ s. Results correspond to one subject in the eyes closed condition.

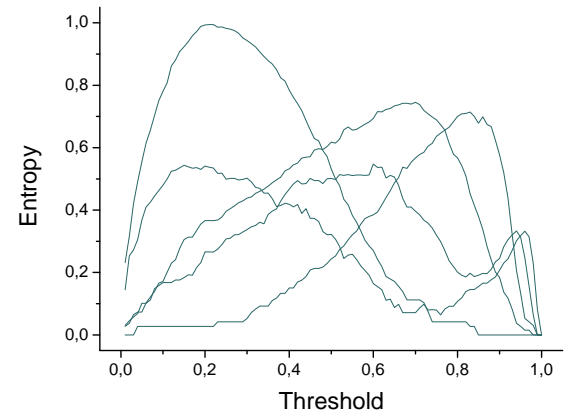

(a)

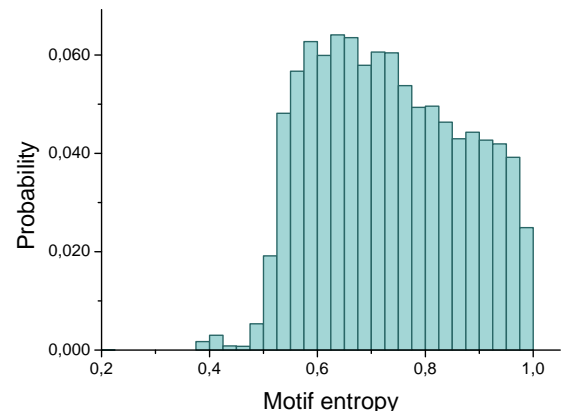

(b)

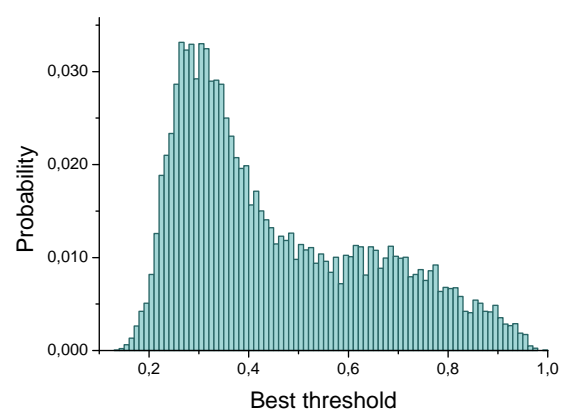

(c)

The most important conclusion that can be drawn from Figure 2 is that each triplet of sensors has to be associated to a different threshold, in order to maximize the information codified in its associated motifs. Furthermore, there is an important variability in such thresholds: while most of the triplets benefit from a low threshold ( $\rho \approx 0.3$ ), a non-neglibible fraction requires $\rho>0.8$.

Table 1. Correlation coefficients and adjusted $R^{2}$ between the best threshold of each triplet, and four explanatory variables. Results correspond to one subject in the eyes closed condition.

\begin{tabular}{lcc}
\hline Explanatory variable & Correlation coefficient & Adjusted $R^{2}$ \\
\hline Average correlation of triplets & 0.5915 & 0.3499 \\
Max correlation in triplets & 0.3449 & 0.11895 \\
Average distance between sensors & -0.7382 & 0.5449 \\
Min distance between sensors & -0.3633 & 0.13194 \\
\hline
\end{tabular}

Figure 3. Scatter plot of the best threshold for each triplet, and the average triplet correlation (a) and distance (b). Results correspond to one subject in the eyes closed condition.

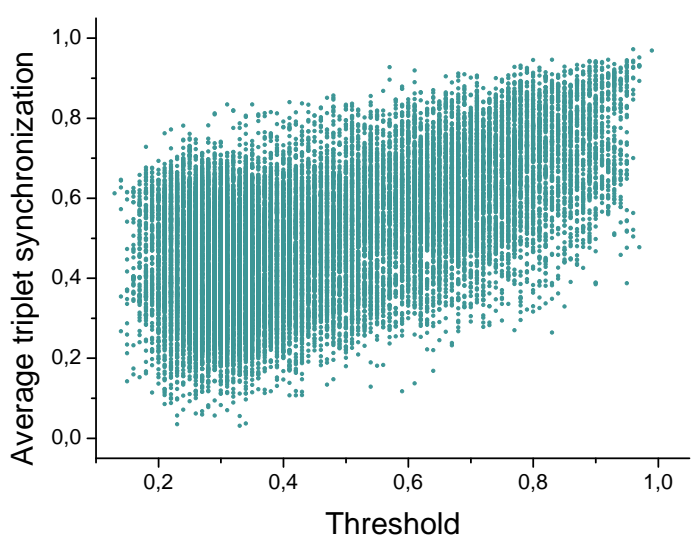

(a)

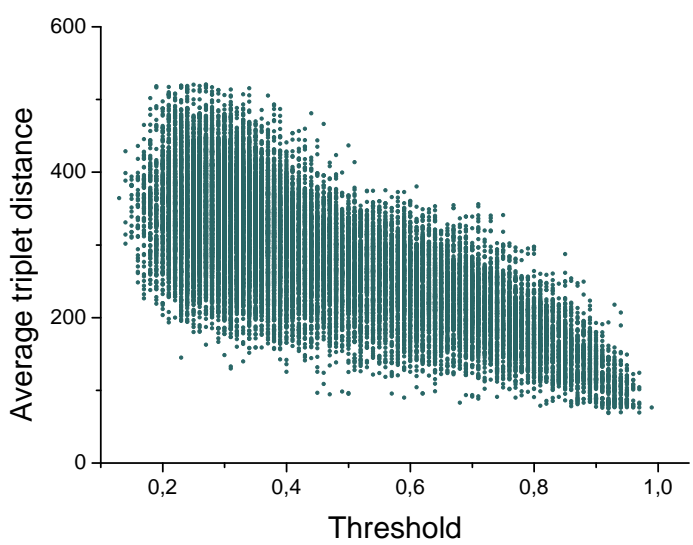

(b) 
It is clear that such variability may be due to volume conduction. Specifically, triplets of neighboring sensors are usually associated with higher correlations; therefore, it may be expected that, in such situations, a higher threshold is required to discriminate the additional synchronization caused by shared information processing. This is confirmed by an analysis of the correlation between the best threshold on the one hand, and the physical distance and synchronization level between the three sensors on the other-see Table 1 and Figure 3.

Figure 4. Topographical maps of relevant triplets of EEG sensors seen from above. Top panels represent the average entropy (a) and threshold (b) of each EEG sensor. In both cases, the selected threshold is the one maximizing the motif entropy. Bottom panels depict the eight triplets with highest entropy (c) and optimal threshold (d). Results correspond to one subject in the eyes closed condition.

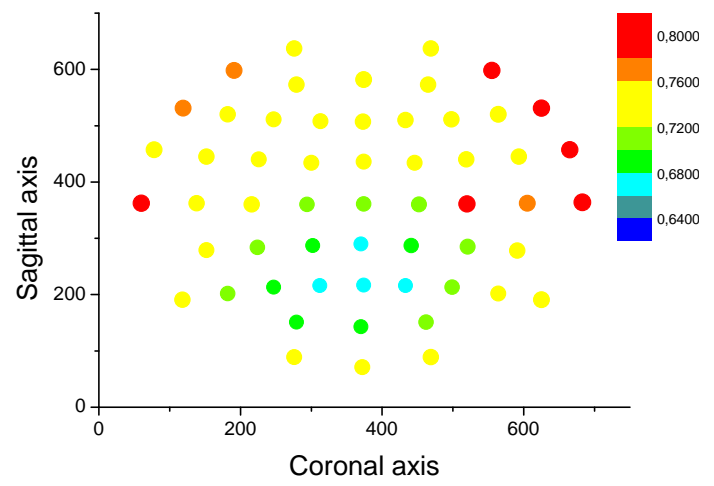

(a)

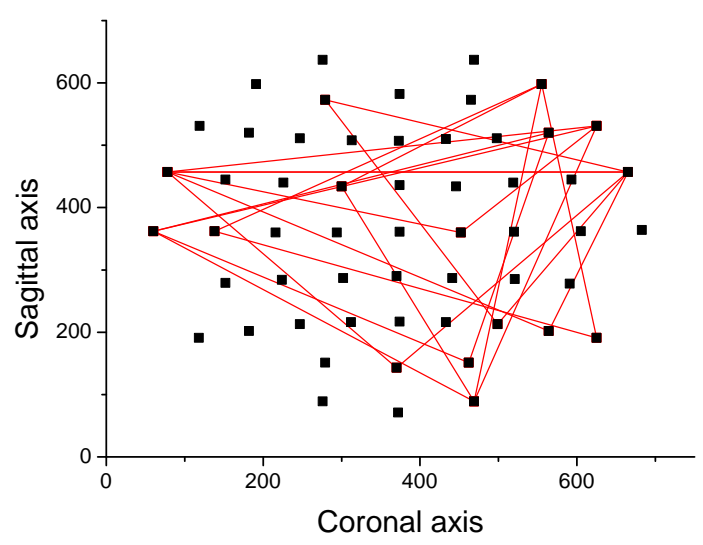

(c)

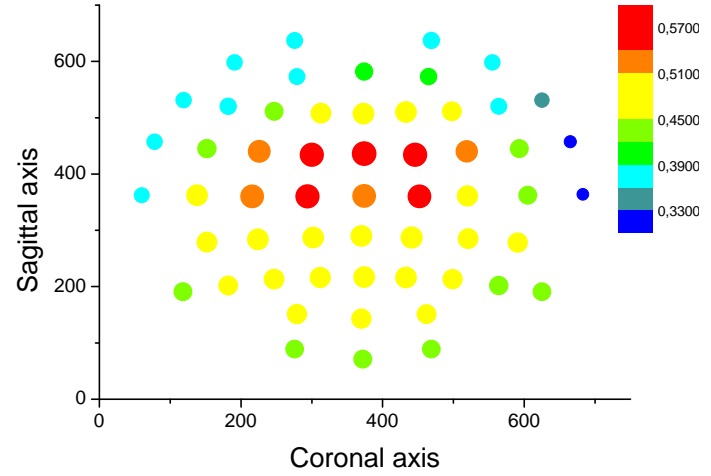

(b)

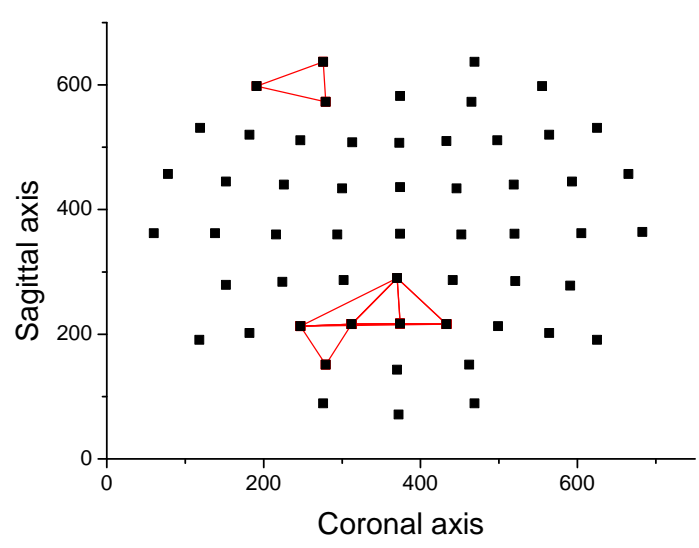

(d)

From an analysis of the correlation results, it appears that only half of the value of the optimal threshold can be explained by volume conduction. In order to explain the source of the other half, Figure 4 depicts the relationship between entropy and the spatial position of nodes. Specifically, the color and size of each dot in Figure 4a,b represent the average entropy and optimal threshold of all triplets to which a given node belongs. Frontal and frontal-temporal electrodes are associated with high entropies and low thresholds, while the opposite holds for parietal electrodes. Additionally, Figure 4c,d report the eight triplets that are associated with the highest entropy and highest threshold. 
Finally, it is worth analyzing if any additional information is obtained by using the number of forbidden motifs, instead of the motif entropy, in order to estimate the best threshold, in a way similar to what usually performed in time series analysis through the Bandt and Pompe methodology [17]. In this case, the optimal threshold corresponds to the one that minimizes the number of forbidden patterns. The correlation between the best thresholds obtained in both cases, 0.5623 , indicates that both approaches are qualitatively similar. Furthermore, a high correlation is obtained between the entropies obtained in both cases, i.e., 0.7781. Due to this similarity, in what follows, only results corresponding to thresholds maximizing the entropy are presented.

\section{Entropy and Time Scales}

Once the best threshold has been defined for each triplet of sensors, it is possible to analyze the evolution of the entropy as a function of the time window length $\tau$. Figure 5a reports such evolution for five triplets of nodes. It can be appreciated that the value of the entropy changes, from low to high $\tau \mathrm{s}$, until an asymptotic value is reached. In order to model this dynamics, the evolution of the entropy of each triplet has been adjusted to the following exponential function:

$$
E=\alpha+\beta e^{-\gamma \tau}
$$

Therefore, $\alpha$ represents the asymptotic entropy value, i.e., the characteristic entropy of the triplet after the initial transient. Furthermore, $\gamma$ represents the characteristic time scale of the triplet, so that the higher the value, the faster the triplet reaches its steady state. Figure 5b,c report the two histograms corresponding to $\alpha$ and $\gamma$ for all triplets of a single subject. A great variability can be observed, especially in the time scale: while most of the triplets have a slow evolution, some of them reach a steady state with a time window of less than 20 samples.

Figure 5. Analysis of time window length. (a) Evolution of the entropy of 5 triplets as a function of the time window length $\tau$, for the optimal threshold (as in Figure 2). (b) Histogram of the asymptotic entropy of each triplet. (c) Histogram of the characteristic time scale of each triplet. Results correspond to one subject in the eyes closed condition. See Equation (2) of the main text for further details.

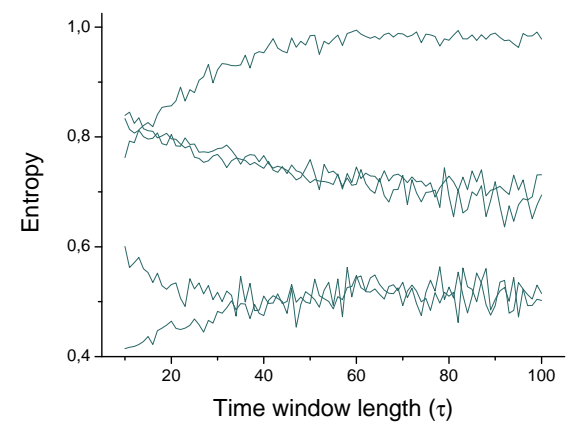

(a)

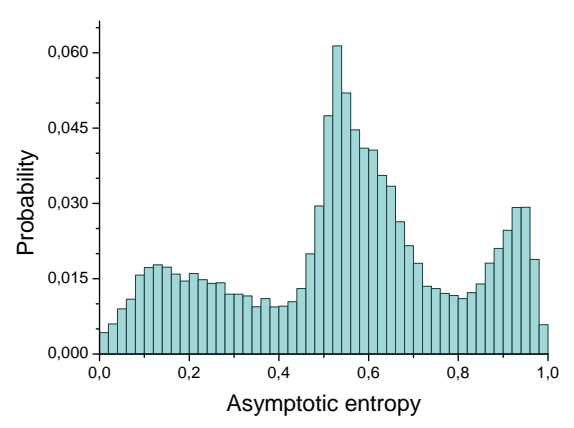

(b)

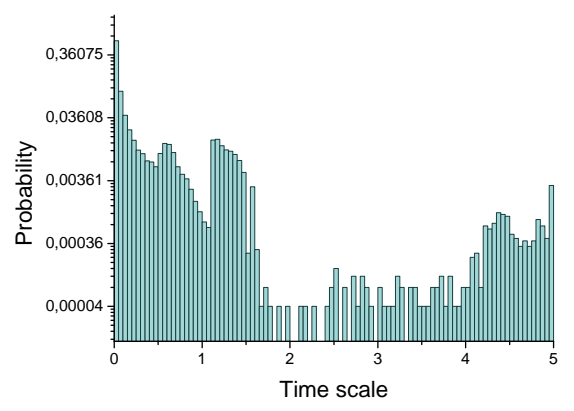

(c)

In a way similar to Figure 4, Figure 6 depicts the spatial information related to the asymptotic entropy and time scale of motifs. Specifically, the color and size of each dot in Figure 6a,b represent the average 
aymptotic entropy and time scale of all triplets in which that given node participates. Furthermore, Figure $6 \mathrm{c}, \mathrm{d}$ report the eight triplets that are associated with the fastest and slowest time scale. As in Figure 4, nodes in the frontal and frontal-temporal regions seems to be associated to a specific dynamics, in this case a very fast time scale. These results are in partial agreement with findings from a recent study using fMRI, where the orbito-frontal limbic module was found to be more dynamic whereas the visual, default mode and somatomotor modules were found to be more static [22]. Additionally, the heterogeneity in the motif time scales suggests that changes in functional connectivity do not occur en masse [22], but instead in an asynchronous manner across the scalp.

Figure 6. Topographical map of relevant triplets. Top panels represent the average asymptotic entropy (a) and of the characteristic time scale (b) of each EEG sensor. Bottom panels depict the eight triplets with highest (c) and lowest (d) asymptotic entropy. Results correspond to one subject in eyes closed condition.

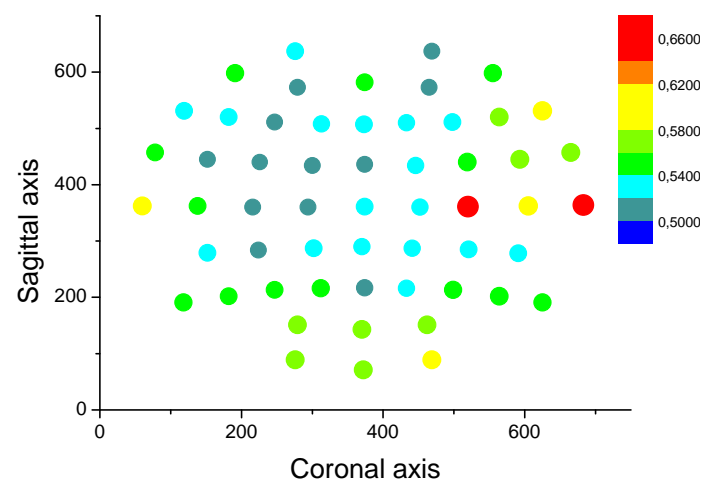

(a)

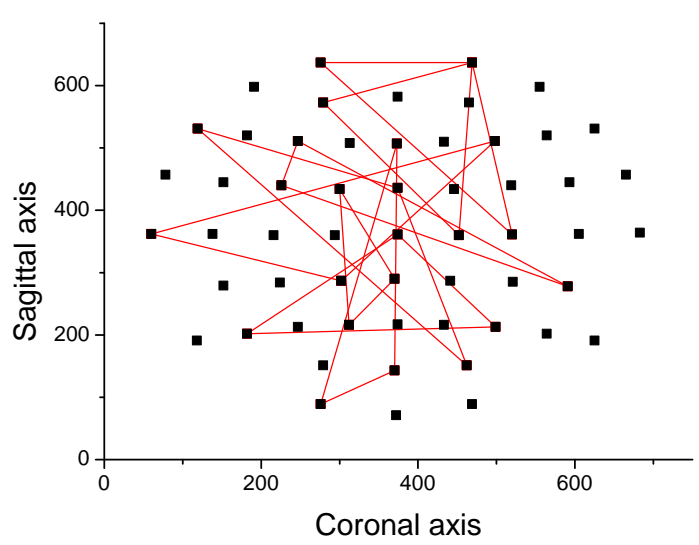

(c)

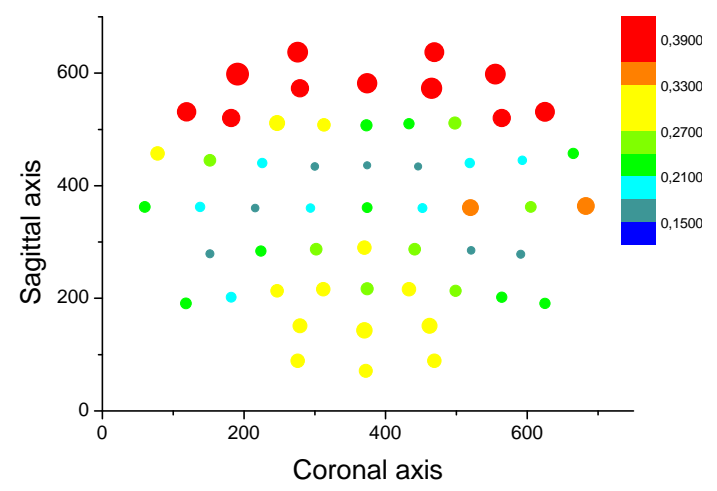

(b)

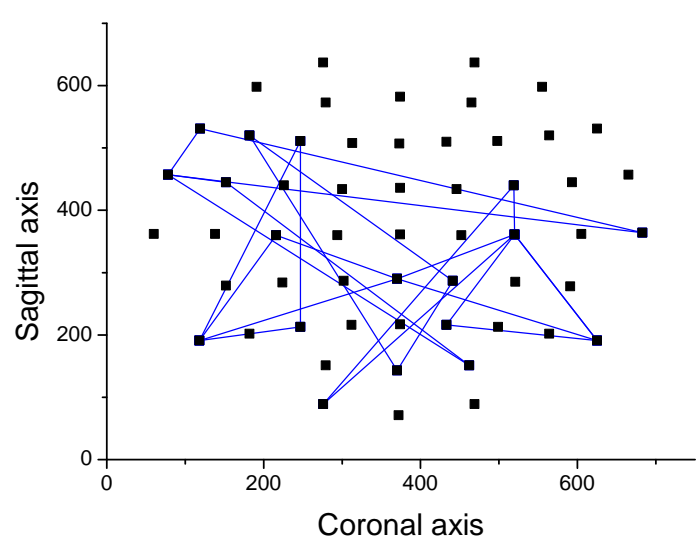

(d)

\section{Extending Motif Entropy}

The concept of motif entropy described here can be extended in several ways: this Section aims at describing some of them, in order to highlight the flexibility and generality of the proposed concept.

First of all, up until now motifs were created using binary values, i.e., links can either exist (whenever the absolute value of the correlation is above a given threshold) or be absent-see Figure 1. When the sign of the correlation is not discarded, the result is the creation of signed motifs [23], i.e., motifs 
whose links can assume three states: $[0,+,-]$. This should, in principle, allow to describe more complex phenomena in brain dynamics, as for instance relay synchronization [24]. The entropy of the symbolic dynamics of these signed motifs can be calculated using Equation (1), taking into account that there are now 27 , as opposed to 8 , possible motifs. Figure 7 a confirms that the frontal region is characterized by a higher entropy, and therefore by a richer dynamics. Furthermore, Figure $7 \mathrm{c}$ presents a scatter plot of the signed motif entropy, as a function of the entropy previously found for each triplet of sensors.

Figure 7. Extending the concept of motif entropy. Top panels represent the average signed motif entropy (a) and motif length entropy (b) for each EEG sensor. Bottom panels depict the relationship between the signed motif entropy (c) and the motif length entropy (d) against the standard motif entropy, for each triplet of EEG sensors. Results correspond to one subject in the eyes closed condition.

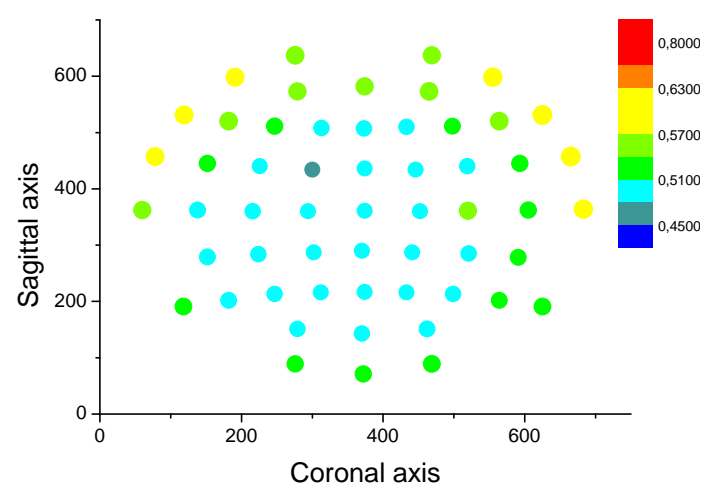

(a)

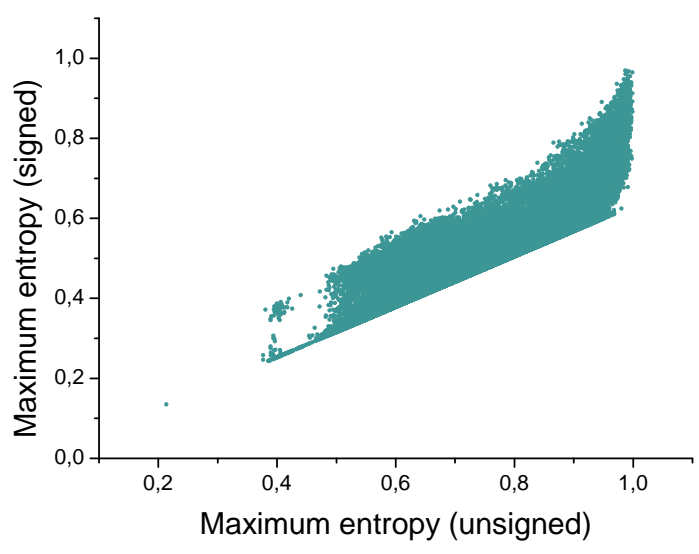

(c)

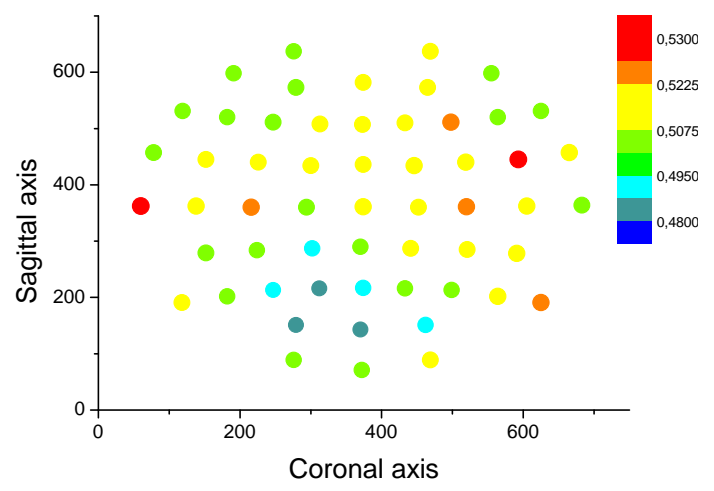

(b)

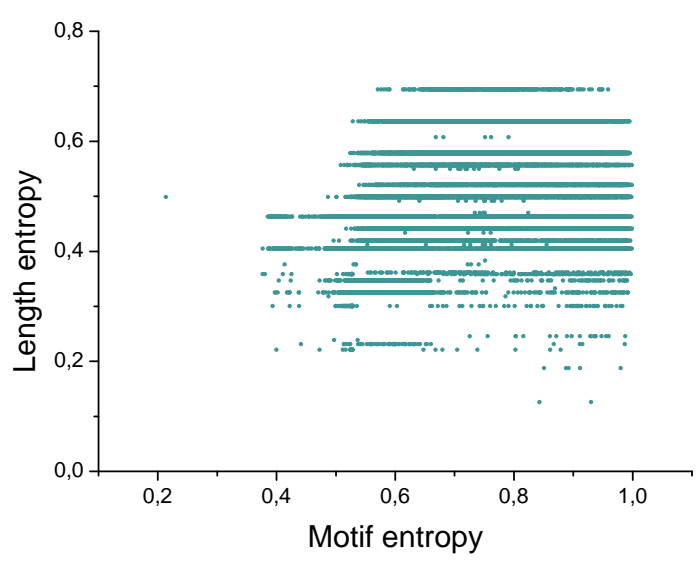

(d)

Besides the probability distribution of motifs, i.e., the probability with which they appear in the time series, one may be interested in the distribution of their lengths, that is, whether they are stable or only appear for short period of times. To this end, one can calculate the average number of times a motif $i$ sequentially appears $\left(l_{i}\right)$, and calculate the associated entropy as:

$$
E_{l}=-\sum_{i} p_{t} \log _{2} p_{t}
$$

$p_{t}$ being the fraction of motifs with $l=t$. The higher $E_{l}$, the more heterogeneous the motifs length: some of them may only appear in short bursts, while other may have a higher stability. Figure $7 \mathrm{~b}$ and $\mathrm{d}$ 
respectively represents the average length entropy for each EEG sensor, and the scatter plot of the length entropy, as a function of the entropy of each triplet of sensors. It can be seen that $E_{l}$ and $E$ are not correlated, indicating that $E_{l}$ provides complementary information about the brain dynamics.

Finally, two other extensions can be analyzed. First, the correlation between consecutive motifs, i.e., the entropy of the matrix $\mathcal{E}$ whose element $(i, j)$ indicates the probability of finding motif $i$ at time $t$ and motif $j$ at time $t+1$. One may also calculate motifs with different number of nodes, e.g., 2, 4, etc. Nevertheless, it should be noticed that the entropy associated with pairs of nodes has little interest, as a threshold can always be found that maximizes the entropy (i.e., that balances the appearance and disappearance of a single link).

\section{Inter- and Intra-Subject Variability}

In order to complete the assessment of the characteristics of the entropy associated to motifs, it is necessary to ascertain the variability associated with each of the described metrics, i.e., optimal thresholds, asymptotic entropy and motif time scales. This may help clarifying two points. On the one hand, if inter-subject variability is low, e.g., the best threshold is constant for all available subjects, this would represent a common feature among different individuals, related with the way the brain processes information. On the other hand, even for high inter-subject variability, a low intra-subject variability would imply that motifs dynamics could be used as a personal fingerprint of the information processing style of each subject.

To address these points, we considered all eight available subjects. Two recordings in two different conditions, eyes opened and closed, were available for each of them, for a total of 16 sets of EEG signals. For each of these sets, the previously described metrics were extracted for all 175.616 triplets of sensors available: optimal thresholds, asymptotic entropy and motif time scales. Inter and intra-subject variability was estimated using the Pearson's linear correlation.

Figure 8 reports the obtained results. Graphs on the left side report the pairwise correlations for the 16 conditions. The two conditions of each subject are grouped together: i.e., the first two rows and columns correspond to subject 1 , the third and fourth to subject 2 , and so forth. The same information is also presented in the right panels, as probability histograms. Top, center and bottom panels respectively correspond to the optimal thresholds, asymptotic entropy and motif time scales.

Two main conclusions can be drawn from Figure 8. Firstly, there is a high inter-subject variability, as shown by the fact that the inter-subject correlations are always below 0.4 both for the asymptotic entropy and the time scales. Interestingly, the inter-subject correlation corresponding to the optimal threshold is significantly high, except for subjects 3 and 8 . As no additional information is available about these two subjects, we cannot know the cause for such difference. Secondly, the intra-subject variability is always lower than the inter-subject one, as indicated by the higher correlation coefficient, suggesting that motifs, and their entropies, may be thought of as subjective brain dynamics fingerprints, irrespective of the condition under which the EEG recording has been made. We note however that these results should be taken with some caution until they are confirmed with a larger population sample and more general experimental conditions. 


\section{Conclusions}

In this contribution, we define a methodology for calculating the entropy corresponding to the dynamics of three-node motifs, based on symbolic analysis. The usefulness of this approach is demonstrated through the study of functional brain activity at rest as measured by EEG. Our results showed each triplet of sensors had a characteristic time scale and synchronization threshold associated with the appearance of functional structure, and that motifs had a topographically specific distribution. Motifs varied widely across subjects, but for each subject, their time scales and thresholds were robust with respect to the experimental condition, suggesting that they may represent a subject-specific dynamical signature.

Figure 8. Analysis of inter-subject variability. Left panels represent the correlation coefficient corresponding to inter- and intra-subjects (see main text for details); correlations between pairs of subjects is codified by the color of the corresponding box, from low (no correlation) to red (perfect linear correlation). On the right panels, the same information is presented in form of histograms. Top, middle and bottom panels respectively correspond to the correlation for thresholds, asymptotic entropy, and motif time scales.
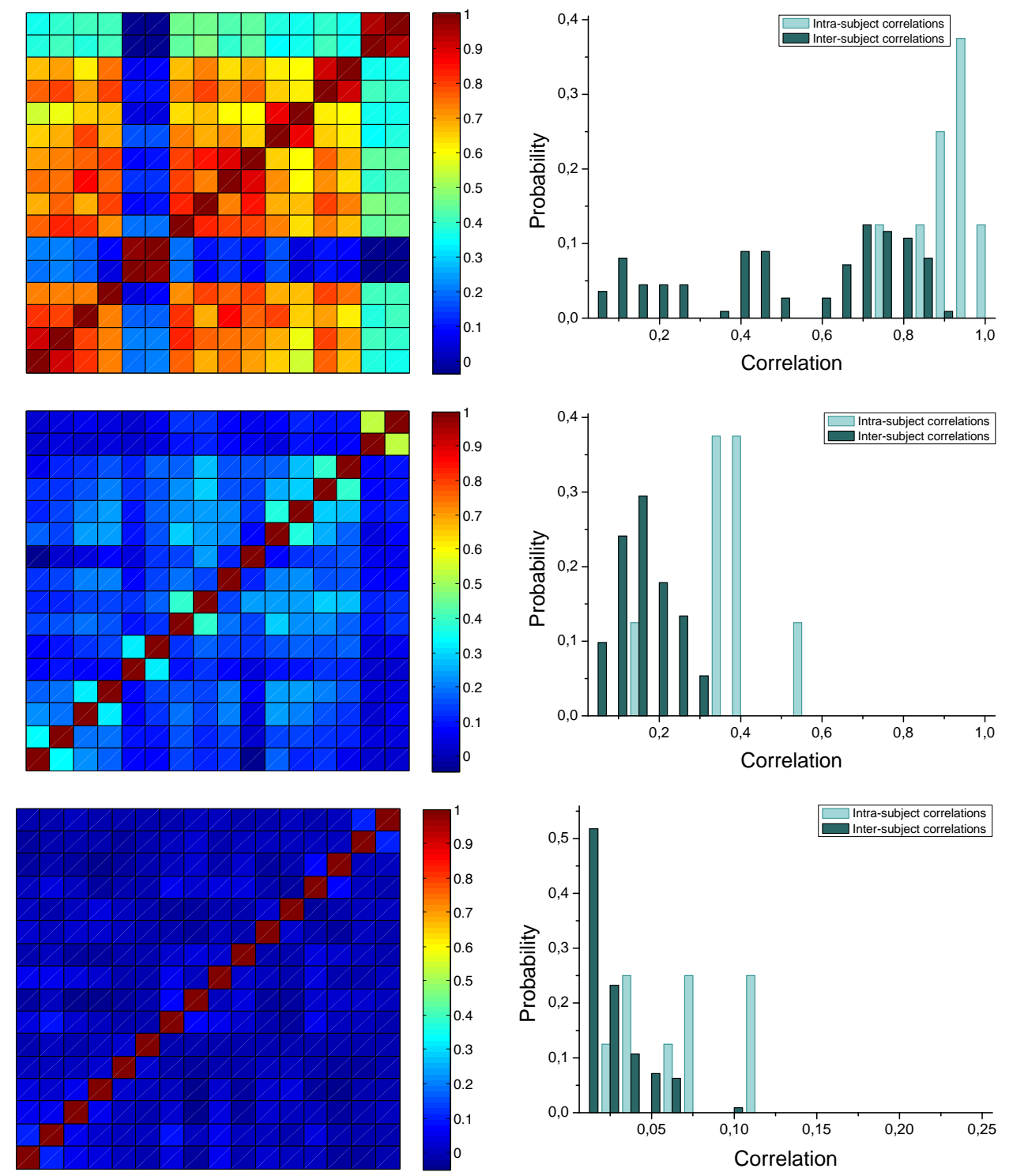
While scalp EEG motifs are not liable to a direct interpretation in computational terms, but only in terms of coordinated activity across brain regions, our method captures the extent to which these patterns are stable in time. Our results suggest that each motif found in the broadband signal of the EEG has its own time scale, sometimes much faster than alpha band ones $(\sim 10 \mathrm{~Hz})$ which dominate resting brain activity, the relationship of which with the oscillation frequency of the component electrodes will need to be clarified.

On the other hand, while the significance of the topographical characteristics of motifs' time scales found in our study should be taken with some caution, given the sample size of our population, the abundance and speed of motifs at frontal electrodes may be consistent with the speficic information integration and executive role of the underlying brain regions.

From a methodological view-point, the time-scale and threshold heterogeneity across motifs indicates that the analysis of motifs cannot be done in a collective way; instead, each motif should be studied separately, using it optimal $\tau$ and $\rho$. More generally, our results point to a complex role of thresholds in network reconstruction. While thresholds are typically chosen once and for all in most network reconstruction studies, each threshold uncovers specific structural aspects of brain activity, so that network analysis should find out the ones that capture fundamental information in a context-specific way.

Finally, the interested reader can find an open-source MATLAB source code for the computation of the motif entropy at [25].

\section{Acknowledgments}

The authors acknowledge the support of MINECO (FIS201238949-C03-01).

\section{Author Contributions}

David Papo developed the original idea. Massimiliano Zanin programmed the software and executed the numerical experiments. Both authors have written the manuscript, read and approved the final manuscript.

\section{Conflicts of Interest}

The authors declare no conflict of interest.

\section{References}

1. Boccaletti, S.; Latora, V.; Moreno, Y.; Chavez, M.; Hwang, D.U. Complex networks: Structure and dynamics. Phys. Rep. 2006, 424, 175-308.

2. Milo, R.; Shen-Orr, S.; Itzkovitz, S.; Kashtan, N.; Chklovskii, D.; Alon, U. Network motifs: Simple building blocks of complex networks. Science 2002, 298, 824-827.

3. Sporns, O.; Kötter, R. Motifs in Brain Networks. PLoS Biol. 2004, 2, doi:10.1371/journal. pbio.0020369.

4. Sakata, S.; Komatsu, Y.; Yamamori, T. Local design principles of mammalian cortical networks. Neurosci. Res. 2005, 51, 309-315. 
5. Song, S.; Sjostrom, P.J.; Reigl, M.; Nelson, S.; Chklovskii, D.B. Highly nonrandom features of synaptic connectivity in local cortical circuits. PLoS Biol. 2005, 3, doi:10.1371/journal.pbio. 0030068.

6. Shen-Orr, S.S.; Milo, R.; Mangan, S.; Alon, U. Network motifs in the transcriptional regulation network of Escherichia coli. Nat. Genet. 2002, 31, 64-68.

7. Mangan, S.; Alon, U. Structure and function of the feed-forward loop network motif. Proc. Natl. Acad. Sci. 2003, 100, 11980-11985.

8. Alon, U. Network motifs: Theory and experimental approaches. Nat. Rev. Genet. 2007, 8, 450-461.

9. Gollo, L.L.; Breakspear, M. The frustrated brain: From dynamics on motifs to communities and networks. Philos. Trans. R. Soc. B 2014, 369, doi:10.1098/rstb.2013.0532.

10. Zanin, M.; Sousa, P.; Papo, D.; Bajo, R.; García-Prieto, J.; del Pozo, F.; Menasalvas, E.; Boccaletti, S. Optimizing functional network representation of multivariate time series. Sci. Rep. 2012, 2, doi:10.1038/srep00630.

11. Holme, P.; Saramäki, J. Temporal networks. Phys. Rep. 2012, 519, 97-125.

12. Boccaletti, S.; Bianconi, G.; Criado, R.; del Genio, C.I.; Gómez-Gardeñes, J.; Romance, M.; Sendiña-Nadal, I.; Wang, Z.; Zanin, M. The structure and dynamics of multilayer networks. Phys. Rep. 2014, 544, 1-122.

13. Crutchfield, J.P.; Packard, N.H. Symbolic dynamics of noisy chaos. Physica D 1983, 7, 201-223.

14. Hao, B.L. Symbolic dynamics and characterization of complexity. Physica D 1991, 51, 161-176.

15. Daw, C.S.; Finney, C.E.A.; Tracy, E.R. A review of symbolic analysis of experimental data. Rev. Sci. Instrum. 2003, 74, 915-930.

16. Bandt, C.; Pompe, B. Permutation entropy: A natural complexity measure for time series. Phys. Rev. Lett. 2002, 88, doi:10.1103/PhysRevLett.88.174102.

17. Zanin, M.; Zunino, L.; Rosso, O.A.; Papo, D. Permutation entropy and its main biomedical and econophysics applications: A review. Entropy 2012, 14, 1553-1577.

18. Gratton, G.; Coles, M.G.; Donchin, E. A new method for off-line removal of ocular artifact. Electroencephalogr. Clin. Neurophysiol. 1983, 55, 468-484.

19. Stam, C.J.; van Dijk, B.W. Synchronization likelihood: An unbiased measure of generalized synchronization in multivariate data sets. Physica D 2002, 163, 236-251.

20. Granger, C.W. Investigating causal relations by econometric models and cross-spectral methods. Econometrica 1969, 37, 424-438.

21. Vicente, R.; Wibral, M.; Lindner, M.; Pipa, G. Transfer entropy-A model-free measure of effective connectivity for the neurosciences. J. Comput. Neurosci. 2011, 30, 45-67.

22. Zalesky, A.; Fornito, A.; Cocchi, L.; Gollo, L.L.; Breakspear, M. Time-resolved resting-state brain networks. Proc. Natl. Acad. Sci. 2014, 111, 10341-10346.

23. Wasserman, S. Social Network Analysis: Methods and Applications; Cambridge University Press: Cambridge, UK, 1994.

24. Fischer, I.; Vicente, R.; Buldú, J.M.; Peil, M.; Mirasso, C.R.; Torrent, M.C.; García-Ojalvo, J. Zero-lag long-range synchronization via dynamical relaying. Phys. Rev. Lett. 2006, 97, doi:10.1103/PhysRevLett.97.123902. 
25. MEntropy source code webpage. Available online: www.mzanin.com/MEntropy (accessed on 27 October 2014).

(c) 2014 by the authors; licensee MDPI, Basel, Switzerland. This article is an open access article distributed under the terms and conditions of the Creative Commons Attribution license (http://creativecommons.org/licenses/by/4.0/). 